\title{
Influência da maturação sexual, idade cronológica e índices de crescimento no limiar de lactato e no desempenho da corrida de 20 minutos*
}

\author{
Deivis Elton Schlickmann Frainer, Fernando Roberto de Oliveira e J oris Pazin
}

\section{RESUMO}

Crianças e adolescentes apresentam, em uma determinada carga, menores concentrações de lactato [la] do que os adultos; especula-se que essas diferenças são ligadas a aspectos maturacionais. O objetivo deste estudo foi verificar a influência da maturação sexual, idade cronológica (I) e índices de crescimento (IC - massa corporal, estatura e somatório de dobras cutâneas subescapular e tricipital) na velocidade do limiar de lactato na concentração fixa de lactato ([la]) de $2,5 \mathrm{mmol} . \mathrm{I}^{-1}\left(\mathrm{~V}_{2,5}\right)$ e na corrida de 20 minutos $\left(V_{20}\right)$. Trinta e três meninos participantes de escolas de esportes foram submetidos a: 1) avaliação antropométrica e avaliação da maturação sexual através dos índices de Tanner (maturação sexual de órgãos genitais e maturação sexual de pêlos púbicos); 2) teste progressivo descontínuo de 3 x 800m (pista de atletismo) para determinar $\mathrm{V}_{2.5}$; e 3) corrida de 20 minutos para determinar a $\mathrm{V}_{20}$ e a [la] final. Não foi encontrada associação entre maturação sexual, idade cronológica e indicadores de crescimento com $\mathrm{V}_{2,5}$. Somente a estatura se mostrou associada com $V_{20}$. Assim, outras variáveis de desempenho, fisiológicas ou biomecânicas, podem influenciar mais no limiar de lactato e na corrida de 20 minutos, do que as variáveis de crescimento durante a adolescência.

\section{ABSTRACT \\ Influence of sexual maturation, chronological age and growth indices in lactate threshold and performance in the $\mathbf{2 0}$ min- utes running}

Children and adolescents present less blood lactate concentrations ([la]) than adults under certain loads. It is suggested that these differences are related to maturational aspects. The aim of this study was to verify the influence of sexual maturation, chronological age and growth indices (body weight, height and sum of two skinfolds - subscapular and triceps) over the lactate threshold velocity, in the fixed lactate concentration of $2.5 \mathrm{mmol}^{-1}\left(\mathrm{~V}_{2.5}\right)$ and in the 20 minutes running $\left(V_{20}\right)$. Thirty-three boys, aged 13-15 years, who practice different sports were submitted to: 1) anthropometrical and sexual maturation evaluations through the Tanner index (sexual maturation of genitals and pubic hair); 2) progressive discontinuous test ( $3 \times 800 \mathrm{~m}$ in running track) to determine $V_{2,5}$; and 3) 20 minutes running test to determine the $V_{20}$ and final blood [la]. There were no associations between $V_{2,5}$ and sexual maturation, neither chronological age nor growth index. As the only association verified was between $V_{20}$ and height $(r=0,41 ; p<0,05)$, the authors conclude that during adolescence other performance,

* Universidade do Estado de Santa Catarina - Florianópolis, Santa Catarina, Brasil, Laboratório de Pesquisa Morfofuncional - LAPEM, Grupo de Pesquisa em Fisiologia e Bioquímica do Exercício.

Recebido em 1/7/05. Versão final recebida em 12/9/05. Aceito em 6/12/05.

Endereço para correspondência: Deivis Elton Schlickmann Frainer, Rua Campolino Alves, 1.199, apto. 406, bloco A, Abraão - 88085-110 - Florianópolis, SC, Brasil. E-mail: frainer_de@yahoo.com.br
Palavras-chave: Maturação sexual. Limiar de lactato. Corrida. Adolescentes.

Keywords: Sexual maturation. Lactate threshold. Running. Adolescents.

Palabras-clave: Madurez sexual. Límite de lactato. Carreras. Adolescentes.

physiological and/or biomechanical variables may play a greater role in lactate threshold and in the 20 minutes running than the growth variables.

\section{RESUMEN}

Influencia de la madurez sexual, edad cronológica e índices de crecimiento en el límite de lactato y en el desempeño en carreras de velocidad de $\mathbf{2 0}$ minutos

Niños y adolescentes presentan en determinada carga menores concentraciones de lactato [la] que los adultos; se especula que esas diferencias están relacionadas a los aspectos de madurez. El objetivo de este estudio ha sido verificar la influencia de la madurez sexual, edad cronológica (I) e índices de crecimiento (IC - masa corporal, estatura y la suma de dobleces cutáneos subescapular y tricipital) en la velocidad del Límite de Lactato en concentración fija de lactato ([la]) de $2,5 \mathrm{mmol} \mathrm{I}^{-1}\left(\mathrm{~V}_{2,5}\right)$ y en carreras de 20 minutos $\left(V_{20}\right)$. Treinta y tres niños participantes de escuelas de deportes fueron sometidos a: 1) evaluación antropométrica y evaluación de madurez sexual a través de los índices de Tanner (madurez sexual de genitales y madurez sexual del pelo pubiano); 2) test progresivo descontinuo de $3 \times 800 m$ (pista de atletismo) para determinar $V_{2,5}, y 3$ ) carreras de 20 minutos para determinar $V_{20} y$ la [la] final. No fue encontrada asociación entre madurez sexual, edad cronológica e indicadores de crecimiento con $V_{2,5}$. Solamente la estatura se mostró asociada con $V_{20}$. Así, otras variables de desempeño, fisiológicas o biomecánicas, pueden influenciar más al final de la concentración de lactato y en las carreras de 20 minutos que las variables de crecimiento durante la adolescencia.

\section{INTRODUÇÃO}

Historicamente, o consumo máximo de oxigênio ( $\mathrm{V}_{2}$ máx) é utilizado como fator determinante do desempenho nas provas de média e longa duração de crianças e adolescentes. No entanto, alguns estudos demonstraram que o $\mathrm{VO}_{2}$ máx não é um bom discriminador do rendimento aeróbio em grupos homogêneos de jovens $^{(1)}$. Com corredores entre 10 e 18 anos, acompanhados longitudinalmente, podem ocorrer melhoras no desempenho de corrida sem modificações correspondentes no $\mathrm{V}_{2}$ máx relativo à massa corporal(2). Além disso, em crianças e adolescentes, nem sempre é possível determinar o $\mathrm{V}_{2}$ máx através do tradicional platô de $\dot{\mathrm{V}}_{2}$, devido principalmente a aspectos maturacionais e dificuldades em se obter um esforço máximo(3). Dessa maneira, é recomendável a utilização de avaliação e prescrição de atividades utilizando modelos submáximos, para atenuar tais dificuldades ${ }^{(4)}$.

Como abordagem padrão de variáveis submáximas, a medida de concentração sanguínea de lactato ([la]) é parte da rotina de 
vários laboratórios de fisiologia do exercício e avaliação funcional. Os chamados limiares de transição, que, basicamente, refletem pontos em que ocorrem aumentos abruptos na curva [la]-intensidade, passaram a ser utilizados como referência de capacidade aeróbia(5). Em verdade, os limiares são aproximações da zona de intensidade no exercício onde ocorreria um equilíbrio entre a produção e a remoção do lactato no sangue, correspondente à intensidade de máximo estado estável de lactato (MEEL). A determinação de limiares, principalmente o limiar de lactato (LL), em alguns trabalhos denominado de limiar anaeróbio (LAn), é utilizada com referências de intensidade para a prescrição das cargas de capacidade aeróbia(6).

Apesar do interesse e evolução tecnológica da medida de [la], esta é uma metodologia custosa e que requer pessoal especializado. Além disso, a coleta de sangue é uma abordagem invasiva e desconfortável, principalmente nos mais jovens. Para atenuar esses problemas metodológicos, diversos autores propuseram alternativas para a predição de variáveis relacionadas ao MEEL e LAn, com abordagens restritas, basicamente, a adultos ${ }^{(7,8)}$, com um número menor de estudos com crianças e adolescentes ${ }^{(9)}$. Com os mais jovens, existe uma deficiência de métodos de estimativa de LAn.

A [la] de 4,0 mmol..$^{-1}$ é freqüentemente utilizada como indicadora do LAn e MEEL em adultos. Entretanto, muitas crianças podem suportar cargas próximas à exaustão sem exceder este valor de [la] ${ }^{10)}$, tornando discutível a sua utilização como critério para avaliar os mais novos. Assim, foram sugeridos o uso de critérios com menores valores como 2,5mmol. $\left.\right|^{-1(10-13)}$.

Cavinato et al. (14), avaliando jovens jogadores de futebol de nível nacional, encontraram [la] de 2,52 $\pm 0,90 \mathrm{mmol}^{-1}{ }^{-1}$, um minuto após uma corrida em esforço máximo constante de $20 \mathrm{~min}$, sendo este valor similar ao sugerido como referência fixa de [la] no LAn $\left(2,5 \mathrm{mmol} . \mathrm{I}^{-1}\right)$ para crianças e adolescentes, demonstrando que a velocidade média neste esforço pode ser uma alternativa para a aproximação do LAn nesses indivíduos.

Pazin et al.(15), com o objetivo de verificar a possibilidade de determinação de velocidades de referência de MEEL (utilizando como critério $2,5 \mathrm{mmol} . .^{-1}$ ) através do teste de corrida de 20 minutos em pista, avaliou 56 jovens participantes de escolas de esportes. Seus resultados mostraram que a velocidade na corrida de 20 minutos não é diferente estatisticamente da velocidade de $2,5 \mathrm{mmol} . \mathrm{I}^{-1}$, concluindo que a corrida em 20 minutos possui bom poder discriminador da aptidão aeróbia de jovens atletas.

Desde os estudos de Ericksson et al.(16-18) vem sendo especulado que as menores [la] nos jovens estariam ligadas a uma menor capacidade glicolítica dos mesmos, e isso estaria relacionado com o processo maturacional. Entretanto, essas evidências vêm sendo questionadas por outros estudiosos e merecem que sejam mais bem investigadas ${ }^{(19)}$. A idade cronológica e os indicadores de crescimento como, por exemplo, peso e estatura, têm sido citados na literatura como possíveis influenciadores do desempenho aeróbio de jovens ${ }^{(1)}$.

Tendo em vista essas evidências, o objetivo deste estudo foi verificar a influência da maturação sexual, idade cronológica e índices de crescimento na velocidade do limiar de lactato e no desempenho da corrida de 20 minutos.

\section{MÉTODO}

\section{Amostra}

A amostra constou de 33 meninos, entre 13 e 15 anos, participantes de Escolas de Esportes (basquetebol, voleibol e atletismo), em clubes e entidades esportivas da região de Florianópolis - SC. A coleta de dados foi realizada após a assinatura de consentimento informado pelos pais, conforme a aprovação do Comitê de Ética e Pesquisa da Universidade do Estado de Santa Catarina.
Medidas antropométricas, idade cronológica comigida e avaliação da maturação sexual

Inicialmente, foram obtidas da amostra a massa corporal, a estatura e a tomada de dobras cutâneas tricipital e subescapular para efetuar o somatório de dobras cutâneas ( $\Sigma D C)$. As medidas foram realizadas de acordo com a padronização de Lohman (1991) citado por Tritschler(20).

A idade cronológica foi corrigida (ICC) pela data de nascimento de cada indivíduo em relação à data de realização da obtenção dos dados antropométricos dos mesmos; esse ajuste se deu em ordem decimal.

A maturação sexual foi determinada através dos índices padronizados por Tanner ${ }^{(21)}$, observando-se o desenvolvimento das $\mathrm{Ca}$ racterísticas sexuais secundárias de pilosidade pubiana (MSPP) e desenvolvimento de órgãos genitais em meninos (MSOG). Os jovens receberam a instrução acerca do significado da avaliação e, em seguida, uma ficha contendo as figuras padronizadas e com um campo ao lado para que eles respondessem a respeito do estágio maturacional que, observando nas figuras, se aproximava ao deles. Esse preenchimento foi realizado de forma individualizada: o adolescente preencheu a ficha em uma sala fechada. Após isso, somente os pesquisadores tiveram acesso aos dados preenchidos pelo jovem.

Os jovens foram agrupados como púberes quando se encontravam nos estágios 2 e 3, tanto de pilosidade púbica quanto de órgãos genitais, e como pós-puberes quando se encontravam nos estágios 4 e 5.

\section{Teste de comida de $\mathbf{2 0}$ minutos}

O teste foi realizado em pista de 200 metros de material sintético, com os jovens realizando um aquecimento de 10 minutos com corrida contínua de baixa intensidade e 10 minutos de exercícios de alongamento; foi recomendado aos participantes que mantivessem a velocidade constante durante a corrida, com o objetivo de percorrerem a maior distância possível em 20 minutos. A freqüência cardíaca (FC) foi monitorada durante todo o teste (registro da FC de 5 em 5 segundos através do freqüencímetro Polar S610itm) para análise da intensidade de esforço. Houve coleta de sangue, para a mensuração das concentrações sanguíneas de lactato [la], um minuto após o final do teste. As variáveis obtidas nesse teste foram a velocidade média na corrida de 20 minutos $\left(V_{20}\right)$, freqüência cardíaca no final do teste $\left(\mathrm{FC}_{\text {final }}\right)$ e percentual da $\mathrm{FC}$ máxima estimada (\% $\mathrm{FC}_{\text {máx }}$ ). $\mathrm{O}$ teste foi realizado em grupos de no máximo cinco indivíduos.

\section{Teste progressivo}

Os jovens realizaram (em pista de atletismo de $200 \mathrm{~m}$ ) três corridas de 800 metros (com um minuto de intervalo), com a intensidade do esforço sendo controlada por zonas de freqüência cardíaca preestabelecidas para cada corrida. Esta metodologia de teste progressivo foi modificada de Geysemeyer e Rieckert'(22). Após um período de aquecimento de 10 minutos com corrida contínua de baixa intensidade e 10 minutos de exercícios de alongamento, foi executada a primeira corrida de $800 \mathrm{~m}$, quando o indivíduo era orientado a manter a FC entre 140 e $150 \mathrm{bpm}$; as outras corridas foram realizadas em valores de FC entre $160-170 \mathrm{bpm}$ e $180-190 \mathrm{bpm}$, controlados a partir do monitor de FC. O intervalo entre cada corrida de 800 metros foi de um minuto. Imediatamente após cada corrida de $800 \mathrm{~m}$, houve coleta de sangue para mensuração das [la]. As medidas das [la] no sangue foram analisadas por método eletroenzimático em um aparelho YSI 1500 (Yellow Springs Instruments ${ }^{\circledR}$ ), com amostras de 25 microlitros de sangue capilar retiradas do lóbulo da orelha. Para a medida da FC utilizaram-se monitores de FC Polar S610 (Polar Electro ${ }^{\circledR}$.

A variável obtida nesse teste foi a velocidade média na [la] de $2,5 \mathrm{mmol}^{-1} \mathrm{I}^{-1}\left(\mathrm{~V}_{2,5}\right)$. Para identificar $\mathrm{V}_{2,5}$ utilizou-se o método de inter- 
polação e extrapolação linear a partir da plotagem das [la] nas suas respectivas velocidades médias obtidas do teste progressivo. $\mathrm{O}$ limite utilizado de extrapolação foi menor ou igual a 0,5mmol..$^{-1}$.

Os testes foram realizados de forma balanceada para diminuir a interferência do seu efeito nos resultados.

\section{Análise estatística}

Os jovens foram divididos em grupos por idade cronológica e por índice maturacional. Para verificar associação entre duas variáveis foi aplicada a correlação simples de Pearson. A correlação de Spearman-Rank foi utilizada para verificar a associação entre a maturação sexual e as demais variáveis do estudo. O comparativo entre os índices de crescimento (peso, estatura e somatório de dobras cutâneas), $V_{2,5}$ e $V_{20}$ nas idades cronológicas se deu através do teste ANOVA, utilizando post-hoc de Scheffé. O comparativo nos estágios maturacionais se deu através do teste $t$ para amostras independentes. A partir das análises foi estabelecido um modelo de regressão para a estimativa de $\mathrm{V}_{20}$ e $\mathrm{V}_{2,5}$, utilizando as seguintes variáveis preditoras: estágio maturacional, idade, gênero, peso, estatura e \% de gordura. ANOVA two-way foi utilizada para comparar $\mathrm{V}_{20}$ e $\mathrm{V}_{2,5}$ entre as diferentes idades cronológicas e estágio maturacional. Para todas as análises foi utilizado o nível de significância para $\mathrm{p}<0,05$.

\section{RESULTADOS}

Os valores de massa corporal, estatura e somatório de dobras cutâneas ( $\Sigma D C)$ para a amostra de meninos de 13, 14 e 15 anos encontram-se na tabela 1 . Apenas a estatura foi diferente comparando as idades de 13 e 15 anos.

\begin{tabular}{lccc}
\hline \multicolumn{4}{c}{$\begin{array}{c}\text { TABELA 1 } \\
\text { Valores (média } \pm \text { desvio-padrão) das variáveis } \\
\text { antropométricas segundo os grupos de idade }\end{array}$} \\
\hline Variáveis & $\mathbf{1 3}$ anos & $\mathbf{1 4}$ anos & $\mathbf{1 5}$ anos \\
N & 13 & 11 & 9 \\
Massa $(\mathrm{kg})$ & $58,66 \pm 14,36$ & $62,89 \pm 17,08$ & $72,46 \pm 15,30$ \\
Estatura $(\mathrm{cm})$ & $166,33 \pm 10,75^{*}$ & $171,29 \pm 9,81$ & $179,63 \pm 5,05$ \\
$\Sigma D C(\mathrm{~mm})$ & $23,19 \pm 10,35$ & $23,57 \pm 9,36$ & $20,53 \pm 11,23$ \\
\hline
\end{tabular}

$\mathrm{N}=$ número de sujeitos; $\Sigma \mathrm{DC}=$ somatório de dobras cutâneas.

* = diferença significante de 15 anos $(p<0,05)$.

Comparando idade cronológica corrigida, massa corporal, estatura e $\Sigma D C$ entre os grupos de jovens púberes e pós-púberes, utilizando, como critério de maturação sexual, a auto-avaliação de órgãos genitais (MSOG), não foram encontradas diferenças significantes em nenhuma variável analisada (tabela 2). J á comparando entre os grupos de jovens púberes e pós-púberes, utilizando como critério de maturação sexual a auto-avaliação da pilosidade púbica (MSPP), foram encontradas diferenças significantes entre massa e estatura (tabela 2).

TABELA 2

Características físicas de púberes e pós-púberes através dos métodos de auto-avaliação da maturação sexual

\begin{tabular}{|c|c|c|c|c|}
\hline \multirow[t]{2}{*}{ Variáveis } & \multicolumn{2}{|c|}{ Órgãos genitais } & \multicolumn{2}{|c|}{ Pilosidade púbica } \\
\hline & Púberes & Pós-púberes & Púberes & Pós-púberes \\
\hline $\mathrm{N}$ & 17 & 16 & 10 & 23 \\
\hline Idade (anos) & $14,25 \pm 0,77$ & $14,35 \pm 0,91$ & $13,92 \pm 0,74$ & $14,47 \pm 0,82$ \\
\hline Massa (kg) & $60,68 \pm 17,97$ & $67,18 \pm 13,60$ & $48,89 \pm 8,17^{*}$ & $70,33 \pm 14,28$ \\
\hline Estatura (cm) & $169,78 \pm 12,61$ & $173,56 \pm 7,46$ & $161,96 \pm 10,38 *$ & $175,81 \pm 7,36$ \\
\hline$\Sigma \mathrm{DC}(\mathrm{mm})$ & $23,72 \pm 10,56$ & $21,39 \pm 9,65$ & $21,96 \pm 7,27$ & $22,87 \pm 11,16$ \\
\hline
\end{tabular}

$\mathrm{N}=$ número de sujeitos; $\Sigma \mathrm{DC}$ = somatório de dobras cutâneas

* diferença significante de pós-púberes $(p<0,005)$.
Comparando as variáveis de performance $\mathrm{V}_{2,5}(2,81 \pm 0,34 ; 2,63$ $\left.\pm 0,36 ; 2,86 \pm 0,45 \mathrm{~m}_{\mathrm{s}} \mathrm{s}^{-1}\right)$ e $\mathrm{V}_{20}(2,79 \pm 0,45 ; 2,80 \pm 0,31 ; 2,97 \pm$ $0,25 \mathrm{~m} . \mathrm{s}^{-1}$ ) nas idades de 13,14 e 15 anos, respectivamente, também não houve diferença significante entre as idades.

Os valores de $V_{2,5}$ e $V_{20}$ entre os grupos de jovens púberes e pós-púberes (MSOG e MSPP) estão representadas nas figuras $1 \mathrm{e}$ 2. Não foram encontradas diferenças significantes entre púberes e pós-puberes para as variáveis de performance $\left(V_{2,5}\right.$ e $\left.V_{20}\right)$ utilizando como critério MSOG; no entanto, utilizando MSPP, foi encontrada diferença significante na $V_{20}(p<0,05)$.

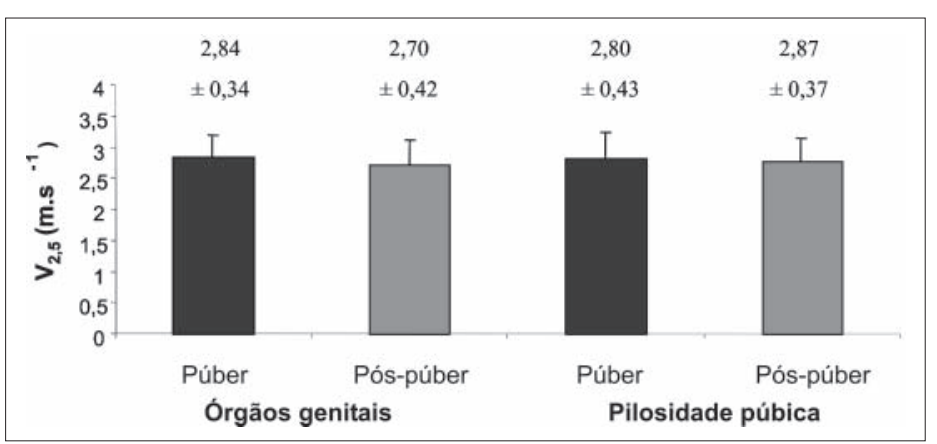

Figura 1 - Comparação entre púberes e pós-púberes na velocidade do limiar de lactato através da auto-avaliação da maturação sexual

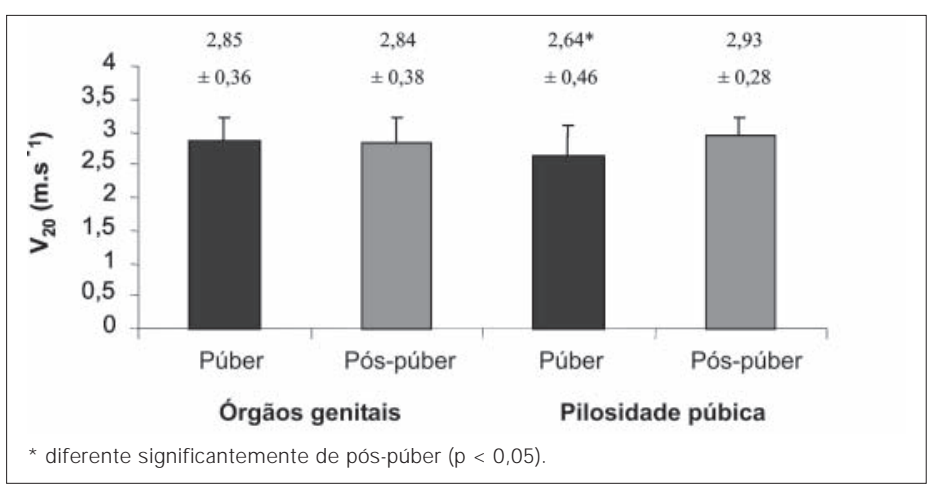

Figura 2 - Comparação entre púberes e pós-púberes na $V_{20}$ através da auto-avaliação da maturação sexual

As correlações entre maturação sexual (MSOG e MSPP), ICC, índices de crescimento, $\mathrm{V}_{2,5}$ e $\mathrm{V}_{20}$ estão presentes na tabela 3.

\section{TABELA 3}

Correlações entre maturação sexual (MSOG e MSPP), idade cronológica corrigida (ICC), índices de crescimento, $V_{2,5} \mathbf{e ~ V}_{20}$

\begin{tabular}{|c|c|c|c|c|c|c|c|}
\hline & MSOG*** & MSPP*** & ICC & Massa & Estatura & $\Sigma D C$ & $\mathbf{V}_{2,5}$ \\
\hline MSPP & ,48* & & & & & & \\
\hline ICC & ,03 & ,35* & & & & & \\
\hline Massa & ,30 & ,70* & ,27 & & & & \\
\hline Estatura & ,07 & ,56* & ,44* &, $75^{* *}$ & & & \\
\hline$\Sigma D C$ &,- 11 & ,02 &,- 07 &, $49 * *$ &, 13 & & \\
\hline $\mathrm{V}_{2,5}$ & ,16 & ,03 & ,05 &, 14 &, 09 & ,30 & \\
\hline $\mathrm{V}_{20}$ &,- 09 & ,31 & ,18 & ,21 & ,37* &,- 07 & ,39* \\
\hline
\end{tabular}

Tanto $\mathrm{V}_{2,5}$, quanto $\mathrm{V}_{\mathrm{T} 20}$, não foram diferentes em relação a diferentes estágios maturacionais (fator 1 ) e idade cronológica (fator 2), utilizando ANOVA two-way.

Realizou-se uma análise de regressão múltipla (stepwise) para entender melhor quais das variáveis estudadas (maturação sexual, índices de crescimento e variáveis de desempenho) foram relevantes na determinação tanto de $\mathrm{V}_{2,5}$ quanto $\mathrm{V}_{20}$. 
Quando colocadas as variáveis de maturação sexual e índices de crescimento, nenhuma delas explicou satisfatoriamente $\mathrm{V}_{2,5}$.

Ao inserirmos os índices de maturação sexual e os índices de crescimento para predizer $\mathrm{V}_{20}$, a única variável que entrou no modelo de predição foi a estatura. A equação gerada foi:

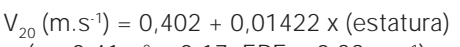

Os resultados da FC final no $T_{20}$ foram de $185 \pm 15 ; 188 \pm 11$; $188 \pm 8 b p m$ nas idades de 13,14 e 15 anos, respectivamente. 0 percentual em relação à FC máxima estimada (220-idade) dos jovens foi $89 \pm 7 \%$; $91 \pm 5 \%$ e $92 \pm 4 \%$.

\section{DISCUSSÃO}

Os resultados dos índices de crescimento, massa corporal e estatura, avaliados nos jovens participantes deste estudo, foram consideravelmente maiores do que os resultados obtidos pelos estudos com escolares catarinenses e escolares da cidade de Florianópolis(23,24).

Também não foi encontrada diferença significante na massa corporal e $\Sigma D C$ entre as idades. Somente foi encontrada diferença significante na estatura entre os jovens de 13 e 15 anos. No entanto, podemos observar que houve uma grande variabilidade nos resultados dos índices de crescimento nessas idades. Essa grande variabilidade pode ser atribuída em parte, segundo Fagundes e $\operatorname{Krebs}^{(24)}$, à associação que existe entre essas variáveis e o período de estirão de crescimento.

Pires e Lopes ${ }^{(23)}$ explicam que o estirão de crescimento é mais tardio e prolongado nos rapazes, em torno dos 14 anos, deixando os rapazes mais altos e mais pesados quando comparados com meninas, que apresentam um estirão mais cedo e de período mais curto, por volta dos 12 anos(25,26). Isso se reflete nas correlações positivas, observadas no presente estudo, entre idade e estatura $(r=0,44)$, massa e estatura $(r=0,75)$, massa e maturação sexual $(r=0,70)$, estatura e maturação sexual $(r=0,56)$ e maturação sexual e idade cronológica $(r=0,35)(p<0,05)$.

Parece que a ação do sistema hormonal, que atua na liberação dos hormônios sexuais e de crescimento, também pode influenciar na variabilidade dos índices de crescimento nas diferentes idades $^{(24)}$.

Esses maiores índices de crescimento observados nos jovens deste estudo podem ser explicados pelo fato de todos os representantes fazerem parte de escolas de esportes da região de Florianópolis nas modalidades de voleibol, basquetebol e atletismo. Todos esses jovens apresentam-se num processo de iniciação esportiva e muitos passaram por "seleções de talentos", em que, nestas modalidades coletivas, as características somáticas são extremamente enfatizadas e relevantes nesse primeiro momento de seleção, o que faz com que a amostra deste estudo esteja muito acima da média dos jovens dos estudos citados acima. Outra possibilidade é o fato de o treinamento regular poder influenciar no crescimento físico e maturação; entretanto, outros fatores podem ter mais relevância na modificação desse processo biológico. Segundo Malina(25), existem interações entre a genética e o ambiente no processo de crescimento e desenvolvimento. Quando as condições ambientais são ótimas, o genótipo é o primeiro regulador do crescimento e maturação. Entretanto, o ambiente social pode influenciar direta ou indiretamente através de fatores como nutrição, as relações familiares, tamanho da família, hábitos de atividade física, hábitos esportivos na família, na escola e na comunidade. Malina ainda conclui que a atividade física, por si só, não determina o crescimento e a maturação. Acredita-se que tal fato deva ter pouco peso nos achados do presente estudo.

As variáveis de desempenho, $V_{2,5}$ e $V_{20}$, também não foram diferentes estatisticamente nas idades de 13, 14 e 15 anos, o que denota uma característica de homogeneidade na capacidade aeróbia dos mesmos. Também não houve associação entre essas variáveis de desempenho e idade cronológica.

Não é possível fazer comparações entre os resultados deste estudo com outros estudos que tiveram objetivos semelhantes. Isso se deve, principalmente, às diferenças metodológicas e conceituais empregadas para caracterizar principalmente a capacidade aeróbia dos jovens. Como exemplo, o estudo de Palgi et al.(27) utilizou o limiar ventilatório e o $\mathrm{VO}_{2 \text { máx }}$ absoluto como indicador de capacidade aeróbia; Tanaka e Shindo(28) utilizaram a velocidade no limiar de lactato abaixo da [la] de $2 \mathrm{mmol}^{-{ }^{-1}}$; Williams et al. utiliza-

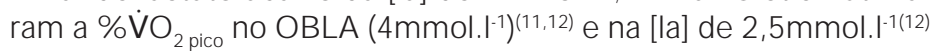
assim como Welsman et al. (29); Armstrong et al. (30) utilizaram como índice de condicionamento cardiorrespiratório o $\dot{\mathrm{VO}}_{2 \text { pico. }}$.

Com o objetivo de verificar a associação entre a maturação sexual e o desempenho em algumas variáveis, utilizaram-se os índices de Tanner(21) através da auto-avaliação do desenvolvimento dos órgãos genitais e do desenvolvimento da pilosidade púbica. A utilização desses índices tem sido recomendada principalmente a estudos transversais( ${ }^{(30)}$ e tem-se constatado uma boa associação com outros indicadores de maturação biológica(25). Entretanto, devese ter cuidado com as análises feitas através desses índices, bem como com a possibilidade de eles estarem discriminando o crescimento e desenvolvimento com acurácia.

Nos resultados deste estudo, levando em consideração a homogeneidade do grupo e o fato de a idade de 13 a 15 anos ser período de diversas modificações nos índices de crescimento, que influenciarão outros componentes de desempenho, e também levando em consideração que existe uma grande variação da idade cronológica para uma mesma idade biológica(31), pode-se observar que a MSOG não discriminou, em nenhuma das variáveis estudadas, o crescimento e desenvolvimento maturacional dos jovens avaliados (vide Resultados - tabelas 2 e 3, e figuras 1 e 2), mesmo não tendo demonstrado diferença significante de MSPP, e os dois índices serem correlacionados significantemente ( $r s=0,48, p<$ $0,05)$. Esses resultados corroboram os encontrados por Borges et al.(32).

Já a MSPP demonstrou diferença entre os jovens púberes e pós-púberes, e associação significante com massa corporal ( $r=$ $0,70)$ e estatura $(r=0,56)(p<0,05)$. Armstrong e Welsman ${ }^{(19)}$ enfatizam que os níveis de testosterona em meninos são altamente correlacionados com os índices de crescimento (massa e estatura) durante a adolescência. Borges et al.(32) recomendam que, quando for empregada a auto-avaliação da maturação sexual para a determinação de eventuais diferenças na aptidão física e nos componentes antropométricos, se utilizem os índices de desenvolvimento da pilosidade púbica como um critério mais eficaz, o que está de acordo com os resultados desse estudo.

Na análise de regressão, os índices de crescimento e a maturação sexual não explicaram o desempenho em $\mathrm{V}_{2,5}$. Parece que em mais jovens, as variáveis de crescimento e a maturação sexual têm baixa associação com o limiar de lactato. Já as variáveis de desempenho de endurance demonstraram maior poder de predição e associação do limiar de lactato, como já fora observado em outros estudos ${ }^{(1,33)}$. Assim, é importante fazer uma reflexão sobre a influencia da maturação sexual no limiar de lactato.

Diversos pesquisadores têm-se preocupado em investigar as razões, a partir dos estudos de Ericksson et al.(16-18), pelos quais os mais jovens apresentavam menores concentrações sanguíneas de lactato. Uma das razões para essa limitação estaria relacionada a uma menor capacidade glicolítica e que conseqüentemente estaria ligada à maturação sexual. Outro estudo, freqüentemente citado pela literatura, demonstrou que para o desenvolvimento da glicólise em ratos é necessária uma proporção adequada de testosterona ${ }^{(34)}$. Falgairette et al.(35) apresentaram associações significantes entre as respostas do lactato sanguíneo no exercício e níveis de testosterona $(r=0,40, p<0,001)$ em 144 meninos de 6 
a 15 anos. Outro estudo clássico que suporta a influência androgênica na glicólise foi realizado por Tanaka e Shindo(28), que demonstraram haver associação significante entre idade óssea e a velocidade no limiar de lactato em meninos de 6 a 15 anos $(r=-$ 0,32 ), concluindo que "meninos pré-púberes e púberes têm maiores velocidades no limiar de lactato do que jovens rapazes (póspuberes), e que isso pode, em parte, ser devido a menor ação da testosterona sobre o músculo esquelético".

No presente estudo, não foi encontrada associação significante entre nenhum dos índices de maturação utilizados (MSOG e MSPP) com $V_{2,5}$ e com $V_{20}$. Esses resultados são similares aos de outros autores que utilizaram tanto os índices de Tanner como marcador de maturação(11,12) quanto as concentrações sanguíneas de testosterona(29).

Vários estudos têm sido criticados por não demonstrarem um controle adequado, confundindo as influências e as intercorrelações entre testosterona, outros índices de crescimento e a variável de desempenho sob consideração. Uma avaliação dos efeitos independentes da testosterona na resposta do lactato sanguíneo deve usar técnicas estatísticas que permitam que essas relações confusas sejam controladas ${ }^{(19)}$.

Em conjunto, parece temerário, à luz do conhecimento atual, assumir sem riscos a relação com os índices de maturação e [la] submáximo/máximo(29) e atividade enzimática glicolítica(36-38). Tanto as metodologias empregadas quanto a magnitude verificada nas associações não dão sustentação suficiente à idéia da dependência, normalmente divulgada, entre a maturação e os limiares de transição. Nos próprios estudos iniciais de Ericksson, que sugerem uma menor habilidade dos jovens em gerar energia pela glicólise, é recomendado pelo próprio autor que haja cautela na interpretação de seus resultados, e, portanto, que "conclusões gerais não podem ser realizadas" (17,p.18).

Diferentemente da inconsistência das evidências de um inferior potencial glicolítico em mais jovens, os estudos têm consistentemente observado elevados níveis de enzimas oxidativas, por exemplo, succinato desidrogenase $(\mathrm{SDH})$ e isocitrato desidrogenase (ICDH)(37). Diferenças na razão entre PFK (fosfofrutoquinase) para ICDH em crianças (0.884) comparado com adultos (1.633) refletem uma melhor habilidade para oxidar o piruvato e prover evidências de que crianças são preferencialmente equipadas para produzir energia aeróbia(38). No entanto, essas considerações não têm efeito comparativo com o presente estudo, pois essas variáveis não foram mensuradas e servem aqui como ponto de reflexão sobre o assunto.

\section{REFERÊNCIAS}

1. Krahenbuhl GS, Skinner J S, Kohrt WM. Developmental aspects of aerobic power in children. Exerc Sport Sci Rev 1985;13:503-38.

2. Daniels J , Oldridge N, Nagle F, White B. Differences and changes in $\mathrm{V}_{2}$ max among runners 10 to 18 years of age. Med Sci Sports Exerc 1978;10:200-3.

3. Armstrong $\mathrm{N}$, Welsman J , Winsley R. Is peak $\dot{\mathrm{VO}}_{2}$ a maximal index of children's aerobic fitness? Int Sports Med 1996;17:356-9.

4. Kiss MAPDM. Potência e capacidade aeróbias: importância relativa em esporte, saúde e qualidade de vida. In: Amadio CA, Barbanti VJ , organizadores. A biodinâmica do movimento humano e suas relações interdisciplinares. São Paulo: Estação da Liberdade, 2000;163-74.

5. Heck H, Hess G, Mader A. Comparative study of different lactate threshold concepts. Dsc ZSportmed 1985;36:19-25.

6. Oliveira FR, Gagliardi J FL, Kiss MAPDM. Proposta de referências para a prescrição de treinamento aeróbio e anaeróbio para corredores de média e longa duração. Rev Paul Educ Fís 1994;8:68-72.

7. Olbrecht J, Madsen O, Mader A, Liesen H, Hollmann W. Relationship between swimming velocity and lactic concentration during continuous and intermittent training exercises. Int J Sports Med 1985;6:74-7.

8. Weltman J , Seip R, Levine S, Snead D, Rogol A, Weltman A. Prediction of lactate threshold and fixed blood lactate concentrations from 3200m time trial running performance in untrained females. Int J Sports Med 1989;10:207-11.
Com relação ao desempenho no teste de corrida de 20 minutos de jovens, só houve associação entre $V_{20}$ e estatura $(r=0,37, p<$ $0,05)$ sendo a única variável com poder de predição em $V_{20}$. Talvez isso seja devido a aspectos de economia de corrida, que ocorrem durante a adolescência, através das modificações nos padrões de freqüência e de tamanho de passada na corrida, devido a alterações no tamanho dos segmentos corporais. É necessário que se analisem outras variáveis que podem ter mais associação com a endurance de jovens, como velocidade máxima de corrida, economia de corrida, fração utilizada do $\dot{V}_{2 \text { máx }}$ (percentual), entre ou$\operatorname{tras}^{(39)}$.

No presente estudo foi solicitado aos jovens que corressem os 20 minutos de forma mais estável possível, sem que houvesse grandes variações do ritmo durante a corrida. Isso foi recomendado por Frainer et al.(40) em um estudo com jovens jogadores de futebol; os autores sugerem cautela ao empregar o teste de 20 minutos e fazer inferências sobre os resultados. O resultado do percentual da freqüência cardíaca máxima (\% $\mathrm{FC}_{\text {máx }}$ ) encontrado no $\mathrm{T}_{20}$ denota que alguns jovens chegaram próximo a $100 \%$ da $\mathrm{FC}_{\text {máx }}$ estimada no final do teste, apresentando altas concentrações de lactato, provavelmente, devido a modificações do ritmo de corrida ao longo dos 20 minutos, proporcionando um desequilíbrio entre a produção e a remoção do lactato sanguíneo ${ }^{(41)}$, podendo prejudicar o desempenho de corrida.

Assim, fica difícil fazer inferências sobre os resultados na corrida de 20 minutos, devido às diferentes estratégias de corrida adotadas pelos jovens nesse estudo.

\section{CONCLUSÃO}

A maturação sexual, a idade cronológica e os índices de crescimento não estão associados com o desempenho na $\mathrm{V}_{2,5^{\circ}}$. Assim, outras variáveis de desempenho, fisiológicas e biomecânicas, podem influenciar mais no limiar de lactato do que as variáveis de crescimento durante a adolescência.

\section{AGRADECIMENTOS}

Agradecemos a todos os integrantes do LAPEM que colaboraram na coleta de dados deste estudo e agradecemos em especial aos professores Lorival J osé Carminatti, Adriano Eduardo Lima-Silva e Ruy J ornada Krebs pelas colaborações e discussões.

Todos os autores declararam não haver qualquer potencial conflito de interesses referente a este artigo.
9. Colantonio E. Análise das velocidades: referencial de $4 \mathrm{mM}$, de equilíbrio de 30 min. E velocidade crítica em nadadores adolescentes. São Paulo, 1999. Dissertação (mestrado) - Escola de Educação Física e Esporte da Universidade de São Paulo.

10. Williams J R, Armstrong N, Kirby BJ. The $4 \mathrm{mmol}$ blood lactate level as an index of exercise performance in 11-13 year old children. J Sports Sci 1990;8:139-47.

11. Williams J R, Armstrong N, Kirby BJ . The influence of age and maturation on the 2.5 and 4.0 mmol levels of blood lactate in girls. J Sports Sci 1990;8:80.

12. Williams J R, Armstrong N, Kirby BJ. The relationship between children's blood lactate responses to incremental and constant speed treadmill exercise. J Sports Sci 1990;8:298-9.

13. Williams J R, Armstrong N, Kirby BJ . The blood lactate response to exercise in 11 to 16 -year-old children with reference to cardiorespiratory variables, chronological age, sex and maturity. J Sports Sci 1990;8:297-8.

14. Cavinato CC, Lima J RP, De Oliveira FR. Teste de 20min e “limiar anaeróbio” em jovens jogadores de futebol. In: IV Congresso Paulista de Educação Física. Anais, J undiaí, 2000.

15. Pazin J , Carminatti L, De-Oliveira FR, Frainer DES, Breda A. Velocidade no teste de corrida de 20 minutos e velocidades de referência de máximo steady-state de lactato. Rev Bras de Ciência e Movimento 2004;1:61. 
16. Ericksson BO, Karlsson J , Saltin B. Muscle metabolites during exercise in pubertal boys. Acta Physiol Scand 1971;87:27-39.

17. Ericksson BO. Physical training, oxygen supply and muscle metabolism in 11-13 years old boys. Acta Physiol Scand 1972;38:1-48.

18. Ericksson BO, Saltin B. Muscle metabolites during exercise in boys aged 11-16 years compared to adults. Acta Physiol Scand 1974;28:257-65.

19. Armstrong N, Wellsman J . Aerobic exercise: growth and maturation. In: Young people \& physical activity. New York: Oxford Univ. Press, 1997.

20. Tritschler K. Medida e avaliação em educação física e esportes de Barrow e McGee. Barueri, SP: Manole, 2003;236-57.

21. Tanner J M. Growth at adolescence. Oxford: Blackwell Scientific, 1962.

22. Geysemeyer U, Rieckert H. Field-step tests for sports discipline-related diagnosis of endurance power. Int J Sports Med 1987;8:132.

23. Pires MC, Lopes AS. Crescimento físico e características sociodemográficas em escolares no município de Florianópolis-SC, Brasil. Rev Bras Cine Des Hum 2004; 6:17-26.

24. Fagundes T, Krebs RJ. Perfil do crescimento somático de escolares de Santa Catarina. Revista Digital: Lecturas en Educación Física, Buenos Aires 2005;10 (83).

25. Malina R. Growth and maturation: normal variation and effect of training. In: Gisolfi CU, Lamb DR, editors. Youth, exercise and sport 1989;223-71.

26. Naughton G, Farpour-Lambert NJ , Carlson J , Bradney M, Van Praag E. Physiological issues surrounding the performance of adolescent athletes. Sports Med 2000;30:309-25.

27. Palgi Y, Gutin B, Young J , Alejandro D. Physiological and anthropometric factors underlying endurance performance in children. Int J Sports Med 1984;5:67-73.

28. Tanaka H, Shindo M. Running velocity at blood lactate threshold of boys ages $6-$ 15 years compared with untrained and trained young males. Int J Sports Med 1985;6:90-4.

29. Welsman J R, Armstrong N, Kirby BJ . Serum testosterone is not related to peak $\dot{\mathrm{VO}}_{2}$ and submaximal blood lactate responses in 12- to 16 -year-old males. Ped Exerc Sci 1994;6:120-7.
30. Armstrong N, Williams J , Balding J , Gentle P, Kirby B. The peak oxygen uptake of British children with reference to age, sex and sexual maturity. EurJ Appl Physiol 1991;6:369-75.

31. Bohme MTS. Resistência aeróbia de jovens atletas mulheres com relação à maturação sexual, idade e crescimento. Rev Bras Cine Des Hum 2004;6:27-35.

32. Borges FS, Matsudo SMM, Matsudo VKR. Perfil antropométrico e metabólico de rapazes pubertários da mesma idade cronológica em diferentes níveis de maturação sexual. Rev Bras de Ciência e Movimento 2004;12:7-12.

33. Mahon AD, Del Corral P, Howe CA, Duncan GE, Ray M. Physiological correlates of 3-kilometer running performance in male children. Int J Sports Med 1996;17: 580-4.

34. Dux L, Dux E, Guba F. Further data on the androgenic dependency of the skeletal musculature. The effect of the prepubertal castration of the structural development of the skeletal muscles. Horm Metab Res 1982;14:191-4.

35. Falgairette G, Bedu M, Fellmann N, Van-Praag E, Coudert J . Bio-energetic profile in 144 boys aged from 6 to 15 years with special reference to sexual maturation. EurJ Appl Physiol 1991;62:151-6.

36. Berg A, Kim SS, Keul J . Skeletal muscle enzyme activities in healthy young subjects. IntJ Sports Med 1986; 7:236-9.

37. Fournier M, Ricci J , Taylor AW, Ferguson RJ , Montpetit RR, Chaitman BR. Skeletal muscle adaptation in adolescent boys: sprint and endurance training and detraining. Med Sci Sports Exerc 1982;14:453-6.

38. Haralambie G. Enzime activities in skeletal muscle of 13-15 years old adolescents. Bull Eur Physiopath Resp 1982;18:65-74.

39. Krahenbuhl GS, Pangrazi RP. Characteristics associated with running performance in young boys. Med Sci Sports Exerc 1983;15:486-90.

40. Frainer DES, De-Oliveira FR, Cal Abad CC, Kiss MAPDM. Evidências de validade do T20 como aproximação do limiar anaeróbio em jovens jogadores de futebol. R. da Educação Física/UEM 2004;15:33-7.

41. Brooks GA. Anaerobic threshold: review of the concept and directions for future research. Med Sci Sports Exerc 1985;17:22-31. 